\title{
Name dominance in spoken word recognition is (not) modulated by expectations: evidence from synonyms
}

Andrea Weber ${ }^{1}$ and Alissa Melinger ${ }^{2}$

${ }^{1}$ Max Planck Institute for Psycholinguistics, The Netherlands

${ }^{2}$ School of Psychology, University of Dundee, United Kingdom

https://doi.org/10.36505/ExLing-2008/02/0057/000116

\begin{abstract}
Two German eye-tracking experiments tested whether top-down expectations interact with acoustically-driven word-recognition processes. Competitor objects with two synonymous names were paired with target objects whose names shared word onsets with either the dominant or the non-dominant name of the competitor. Non-dominant names of competitor objects were either introduced before the test session or not. Eye-movements were monitored while participants heard instructions to click on target objects. Results demonstrate dominant and non-dominant competitor names were considered for recognition, regardless of top-down expectations, though dominant names were always activated more strongly.
\end{abstract}

Key words: spoken-word recognition, synonyms, pre-exposure, eye tracking

\section{Introduction}

Eye movements to displayed objects are one of the most informative methods for studying spoken-word recognition as it happens. Eye-movement studies have, for example, confirmed phonological cohort activation (e.g., Allopenna et al., 1998): when hearing the name of an object, for instance candle, listeners fixate phonologically similar objects like candy more than phonologically dissimilar objects. Cohort activation is furthermore modulated by lexical frequency (e.g., Dahan et al., 2001, Weber and Crocker, 2006): high-frequency cohort competitors are fixated more than low-frequency ones. Semantic information from preceding contexts also constrains cohort activation (e.g., Dahan and Tanenhaus, 2004): listeners no longer fixate competitor pictures when a preceding verb constrains upcoming material. Thus, upon hearing the woman lights the..., only suitable objects like candles but not candy will be fixated. The present eyemovement studies investigated the interaction of bottom-up frequency effects with top-down context effects in German.

Two important changes were incorporated compared with previous studies. Rather than varying lexical frequency between different conceptual objects (e.g., comparing high-frequency competitor bed with low-frequency competitor bell), and thereby introducing the possibility that items vary along dimensions other than frequency, we investigated name dominance effects for single conceptual and pictorial representations. To this end, we

ExLing 2008: Proceedings of 2nd Tutorial and Research Workshop on Experimental Linguistics, 25-27 August 2008, Athens, Greece 
selected competitor objects with two synonymous names that varied in dominance (e.g., 'accordion', dominant Akkordeon and non-dominant Ziehharmonika in German). Some pictorial displays of synonyms can potentially bias towards a particular word form, thereby surpassing lexical frequency effects (e.g., the picture of a magician and a wizard can look differently). We avoided such items, and rather established via a naming pretest that synonym pictures evoked two names with varying frequency. Secondly, testing the influence of word expectation within a sentence context usually complicates effect localization; we therefore modulated listeners' expectations with pre-exposure to a particular word form in isolation. In particular, listeners were introduced to the non-dominant names of competitor pictures prior to the eye-tracking session.

If lexical frequency effects operate for synonyms as they do for other words, more looks to the same competitor picture should be observed when its dominant name is phonologically related to the target picture (Akkordeon for target Akrobat, 'acrobat') than when its non-dominant name is (Ziehharmonika for target Zielscheibe, 'target disc'). If pre-exposure further modulates listeners' expectations for non-dominant competitor names, either by inhibiting high-frequency names or by priming low-frequency names, the magnitude of the competitor activation should vary with pre-exposure.

\section{Experiment \\ Method \\ Participants}

Sixty-four native speakers of German, all students at Saarland University, took part in the experiment for monetary compensation. Half of them participated in Experiment 1a (target similar to non-dominant competitor name), the other half in Experiment $1 \mathrm{~b}$ (target similar to dominant competitor name).

\section{Materials}

Twenty competitor pictures with two synonymous names varying in dominance ('accordion', Akkordeon and Zieharmonika in German) were displayed together with target pictures that were phonological related to either the non-dominant competitor name ('target disc', Zielscheibe, Experiment 1a) or the dominant competitor name ('acrobat', Akrobat, Experiment 1b). Two unrelated distractor pictures were added to each display (e.g., 'pear', Birne, and 'church', Kirche, see Figure 1). Forty filler trials similar in setup but with no phonemic overlap between object names were prepared in addition. 


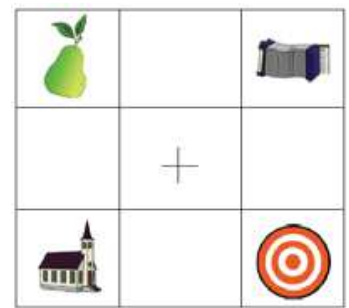

Figure 1. Example display from Experiment 1a.

\section{Procedure}

Instructions to click on objects (e.g., 'Click on the target disc') were presented auditorily. Trials were divided into two blocks. For one test block, the complete set of pictures was shown to the participants before the experiment; for the other block, participants were additionally familiarized with the picture names (non-dominant names for competitor objects).

Listeners' eye movements were monitored while they were listening to the instructions. A camera on the participants' dominant eye provided the input to the eye tracker (SMI Eyelink head-mounted). Onset and offset times and spatial coordinates of eye fixations were recorded.

$1 \mathrm{a}$

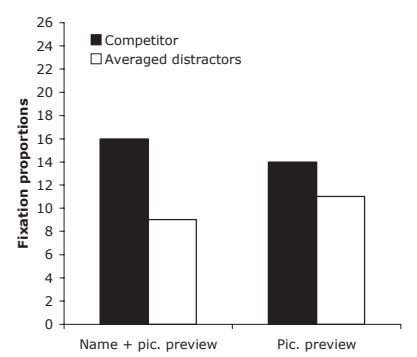

$1 b$

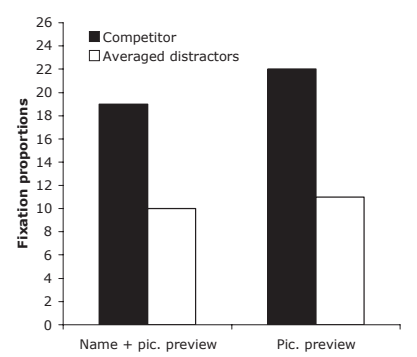

Figure 2. Average fixation proportions for competitor and distractor pictures between 250 and $700 \mathrm{~ms}$ after target onset in Experiments 1a and 1b.

\section{Results}

Figure 2 presents the averaged fixation proportions for cohort competitors and distractors between 250 and $700 \mathrm{~ms}$ after target word onset in Experiments 1a and $\mathrm{b}$. In this time window competitor activation is commonly observed in eye-tracking studies.

When no name expectation was induced in the picture pre-view, listeners looked at the competitor more often when the target was phonologically similar to the dominant name (Exp. 1b) than the non-dominant name (Exp. $1 \mathrm{a} ; \mathrm{t}(62)=-2.13, \mathrm{p}<.04)$, thus replicating previous frequency effects. However, when we biased listeners to expect the non-dominant name, this 
difference disappeared $(\mathrm{t}(62)=-.731, \mathrm{p}>.4)$, suggesting that pre-exposure increased the availability of the non-dominant name. However, the size of the cohort effect was not significantly modulated by expectation. While there was a numerical decrease in the looks to competitors when targets were phonological neighbours of the dominant name, this difference was not statistically reliable, suggesting that pre-exposure does not restrict the candidate set considered by the word recognition system.

\section{Discussion}

Eye movements showed that, indeed, dominant synonym names were activated more strongly than non-dominant synonym names. This finding is in line with previous results for nouns varying in lexical frequency, and generally supports the claim that long-term accrued experience with a word influences the recognition process (e.g., Goldinger, 1998). However, while we did find evidence that pre-exposure increases the availability of the primed name, we did not find evidence that it inhibited the accessibility of the alternative name. This result contrasts recent eye-tracking studies who found an early effect of sentential context on spoken-word recognition. Rather it suggest that lexical access is initially form-based and contextindependent (e.g., Norris et al., 2000).

\section{References}

Allopenna, P. D., Magnuson, J. S., and Tanenhaus, M. K. 1998. Tracking the time course of spoken word recognition using eye movements: evidence for continuous mapping models. Journal of Memory and Language, 38, 419-439.

Dahan, D. and Tanenhaus, M. K. 2004. Continuous mapping from sound to meaning in spoken-language comprehension: immediate effects of verb-based thematic constraints. Language and Cognitive Processes, 30, 498-513.

Dahan, D., Magnuson, J., and Tanenhaus, M. 2001. Time course of frequency effects in spoken-word recognition: Evidence from eye movements. Cognitive Psychology, 42, 317-367.

Goldinger, S.D. 1998. Echoes of echoes? An episodic theory of lexical access. Psychological Review, 105, 251-279.

Norris, D., McQueen, J. M., and Cutler, A. 2000. Merging information in speech recognition: feedback is never necessary. Behavioral \& Brain Sciences, 23, 299 370 .

Weber, A. and Crocker, M. W. 2006. Top-down anticipation versus bottom-up lexical access: Which dominates eye movements in visual scenes. 19th Annual CUNY Sentence Processing Conference, New York. 